\title{
Is There Any Common Pathophysiology between Central Nervous System Lupus and Parkinson's Disease?
}

\author{
Oh-Dae Kwon $^{a}$ Seong-Kyu Kim ${ }^{b}$ Gi-Young Park ${ }^{c}$ \\ a Department of Neurology, School of Medicine, Catholic University of Daegu School of Medicine, Daegu, South \\ Korea; ${ }^{b}$ Department of Rheumatology, School of Medicine, Catholic University of Daegu School of Medicine, Daegu, \\ South Korea; ' Department of Rehabilitation, School of Medicine, Catholic University of Daegu School of Medicine, \\ Daegu, South Korea
}

Dear Sir,

A 51-year-old woman visited the rheumatology department for multiple arthralgia and gait disturbance persisting 11 months. Laboratory tests showed positive fluorescent antinuclear antibody (1:320), Anti-Ro, Anti-Ra, Anti-ds deoxyribonucleic acid immunoglobulin $G$ antibody; and she was diagnosed with systemic lupus erythematosus (SLE) and treatment with prednisolone $10 \mathrm{mg}$ per day was started. The arthralgia improved slowly but the gait disturbance persisted. Neurological examination showed masked face, right dominant rigidity on extremities, decreased arm swing, and slurred gait. Modified Hoehn Yahr scale stage was 3. Fluorine-18 [18F] FP-CIT positron emission tomography (PET) showed decreased uptake of radionucleotide in dorsoposterior portion of both putamen, severe in left which was concurrent with Parkinson's disease (Fig. 1). Accompanied brain computed tomography scan showed no significant abnormality. Levodopa therapy improved the movement symptoms very much. After 2 weeks of levodopa treatment, her gait disturbance worsened again. Brain MRI showed multiple cortical lesions in the left frontoparietal lobe, right parietotemporal lobe, right insular, left cerebellar hemisphere, left frontal lobe, and right parietal lobe with hyperintensity by diffusion imaging (Fig. 1). On state Unified Parkinson's Disease Rating Scale motor score was 76 and modified Hoehn Yahr scale stage was 5. Mini Mental Status Examination score was zero and the mental status deteriorated to stupor state. Cerebrospinal fluid study showed red blood cells (100) without white blood cells and all infection study showed negative. Ribosomal P antibody was positive. She was diagnosed with central nervous system (CNS) lupus and was treated with intravenous steroid pulse therapy (1,000 mg methylprednisolone daily for 3 days) followed by cyclophosphamide, azathioprine, and chloroquine [1]. The symptoms slowly improved and she could walk by herself after 2 months.

As we know, there is only one report introducing anti-dopaminergic antibodies in a patient with a complex autoimmune disorder, rapidly progressing to Parkinson's disease [2]. However, the case did not have dopamine transporter imaging. Coincidence of these 2 diseases in the current case may be explained as follows. Firstly, Parkinson's disease may have an autoimmune base in pathogenesis like SLE [3]. Olfactory dysfunction is quite common in Parkinson's disease, Alzheimer's disease, autism, and CNS lupus. Smelling deficit is closely related with the immune system and there maybe interactions between the smell and the immune system. Therefore, coincidence of Parkinson's disease and CNS lupus could be possible. Secondly, polyadenylation in ribonucleic acid (RNA) processing step may affect both Parkinson's disease and SLE [4]. Polyadenylation is important in messenger RNA stability and deficit in this process may lead to the development of diseases. SLE has polyadenylation defect in GIMAP5 gene and Parkinson's disease has defect in alpha-Synuclein gene. However, a health insurance database in Taiwan of SLE compared incidence rate of Parkinson disease in the SLE registrants with that of age- and sex-matched non-SLE patients. They observed an inverse association between a diagnosis of SLE and the risk of subsequent Parkinson disease, with the crude hazards ratio (HR) being 0.60 and adjusted HR being 0.68 .

\section{KARGER}

(c) 2018 S. Karger AG, Basel
Dr. Oh-Dae Kwon

Department of Neurology, Daegu Catholic University Medical Center School of Medicine, Catholic University of Daegu, 17-33, Duryugongwon-ro Nam-gu, Daegu 42472 (South Korea)

E-Mail dolbaeke@cu.ac.kr 

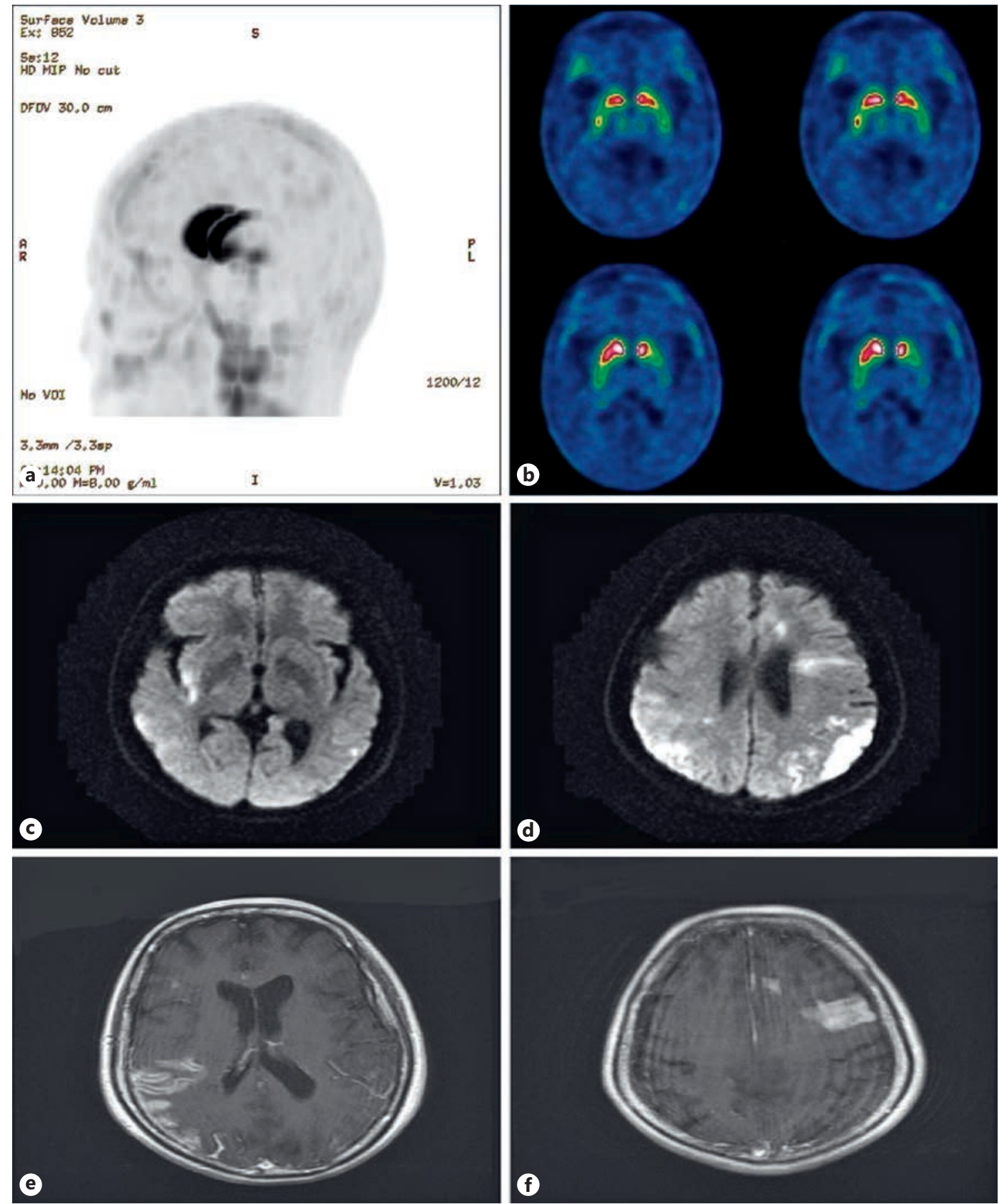

Fig. 1. [18F] FP-CIT PET showed decreased uptake of dopamine transporter in basal ganglia. The 3-dimensional view (a) showed posterior deficit of both putamen. Axial view (b) showed severe deficit in left putamen. Brain MRI showed multiple cortical and white matter lesions in right insular (c), both parietal lobe (d), and enhancing lesions in right parietal cortex (e) and frontal cortex (f). 
This study has critical limitations. They did not clarify diagnostic criteria of PD and the risk factors of PD were not adjusted. Moreover, they did not differentiate the SLE patients with CNS involvement from those without CNS involvement. Therefore, further well-designed epidemiological research is warranted [5].

We introduced a patient diagnosed with CNS lupus and Parkinson's disease at

\section{References}

1 Chiewthanakul P, Sawanyawisuth K, Foocharoen C, Tiamkao S: Clinical features and predictive factors in neuropsychiatric lupus. Asian Pac J Allergy Immunol 2012;30:5560.

2 Kunas RC, McRae A, Kesselring J, Villiger PM: Antidopaminergic antibodies in a patient with a complex autoimmune disorder and rapidly progressing Parkinson's disease. J the same time. The diagnosis of Parkinson's disease was supported by the dopamine transporter imaging and good levodopa response. In conclusion, when we manage patients with Parkinson's disease showing rapidly progressive cognitive symptoms, an autoimmune disease involving the CNS could be a possible consideration in case of prominent history of autoimmune disease. Finding a possible autoimmune mechanism in the development of Parkinson's disease should be done by pathological and immunological methods.

\section{Disclosure Statement}

The authors have no disclosures relevant to the manuscript.
Allergy Clin Immunol 1995;96(5 pt 1):688690.

3 Moscavitch SD, Szyper-Kravitz M, Shoenfeld Y: Autoimmune pathology accounts for common manifestations in a wide range of neuro-psychiatric disorders: the olfactory and immune system interrelationship. Clin Immunol 2009;130: 235-243.
4 Curinha A, Oliveira Braz S, Pereira-Castro I, Cruz A, Moreira A: Implications of polyadenylation in health and disease. Nucleus 2014; 5:508-519.

5 Liu FC, Huang WY, Lin TY, Shen CH, Chou YC, Lin CL, et al: Inverse association of Parkinson disease with systemic lupus erythematosus: A Nationwide Population-based Study. Medicine (Baltimore) 2015;94:e2097. 